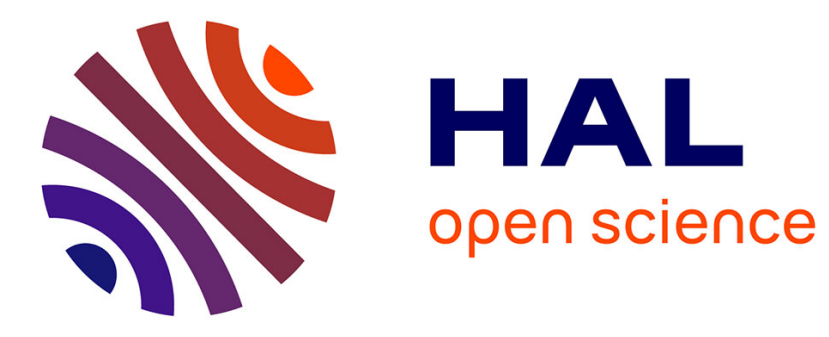

\title{
Joint trellis-coded quantization watermarking for JPEG2000
}

Dalila Goudia, Marc Chaumont, William Puech, Naima Hadl Said

\section{To cite this version:}

Dalila Goudia, Marc Chaumont, William Puech, Naima Hadl Said. Joint trellis-coded quantization watermarking for JPEG2000. Annals of Telecommunications - annales des télécommunications, 2012, 67 (7-8), pp.407-421. 10.1007/s12243-011-0280-9 . lirmm-00805728

\section{HAL Id: lirmm-00805728 https://hal-lirmm.ccsd.cnrs.fr/lirmm-00805728}

Submitted on 28 Mar 2013

HAL is a multi-disciplinary open access archive for the deposit and dissemination of scientific research documents, whether they are published or not. The documents may come from teaching and research institutions in France or abroad, or from public or private research centers.
L'archive ouverte pluridisciplinaire HAL, est destinée au dépôt et à la diffusion de documents scientifiques de niveau recherche, publiés ou non, émanant des établissements d'enseignement et de recherche français ou étrangers, des laboratoires publics ou privés. 


\title{
Joint trellis-coded quantization watermarking for JPEG2000 images
}

\author{
Dalila Goudia • Marc Chaumont • William Puech • \\ Naima Hadj Said
}

Received: 7 March 2011 / Accepted: 22 November 2011 / Published online: 21 December 2011

(C) Institut Télécom and Springer-Verlag 2011

\begin{abstract}
Watermarking in the Joint Photographic Experts Group (JPEG)2000 coding pipeline is investigated in this paper. A joint quantization and watermarking method based on trellis-coded quantization (TCQ) is proposed to reliably embed data during the quantization stage of the JPEG2000 part 2 codec. The central contribution of this work is the use of a single quantization module to jointly perform quantization and watermark embedding at the same time. The TCQbased watermarking technique allows embedding the watermark in the detail sub-bands of one or more resolution levels except the first one. Watermark recovery is performed after image decompression. The performance of this joint scheme in terms of image quality and robustness against common image attacks was estimated on real images.
\end{abstract}

Keywords Image compression • Digital watermarking $\cdot$ JPEG2000 • TCQ

D. Goudia $(\varangle) \cdot$ M Chaumont $\cdot$ W. Puech

Laboratory LIRMM, UMR CNRS 5506,

University of Montpellier II, 161, rue Ada,

34095 Montpellier cedex 05, France

e-mail: dalila.goudia@lirmm.fr

D. Goudia $\cdot$ N. Hadj Said

University of Science and Technologies of Oran (USTO),

BP 1505 El Mnaouer, Oran, Algeria

M. Chaumont

University of Nîmes, rue du Docteur Georges Salan, 30021,

Nîmes cedex 1, France

\section{Introduction}

Digital watermarking is a relatively young research field. Watermarking techniques embed an invisible message within a multimedia content by modifying the media data. This process is done in such a way that the hidden data are not perceptible to an observer and should be resistant to a variety of manipulations. Modeling watermarking as communications with side information has led to the design of very efficient algorithms. This generation of watermarking schemes is called informed schemes [1-3]. One of the two main informed watermarking categories is the quantized-based watermarking schemes. In 1999, Chen and Wornell [1] have introduced the quantization index modulation (QIM), where the host signal is considered as the side information of Costa's scheme [6]. A practical and efficient implementation of the Costa's ideas is the scalar Costa scheme proposed by Eggers et al. [2] which is quiet similar to the DC-QIM watermarking [1].

In many applications, watermarked images are usually compressed in a specific image format before transmission or storage. The simplest way of watermarking is to embed data first with a known watermarking encoder and then compress the watermarked images via a standard compression encoder to get compressed watermarked images. However, the drawback of this approach employing separated watermarking and compression methods is that the compression stage could remove some embedded data from the watermarked images and thus degrade or damage the robustness of the watermark. The compression is considered as an attack. Therefore, instead of treating watermarking and compression separately, it is interesting and beneficial to look at the joint design of watermarking 
and compression system. The joint approach consists to directly embed the binary message during the compression process. The watermarking process is adapted and integrated into the compression coding framework. The main constraints that must be considered are trade-offs between compression bitrate, computational complexity, watermarking payload, distortion induced by the insertion of the watermark, and robustness of watermarked images. As many images are usually compressed by Joint Photographic Experts Group (JPEG)2000 in several applications such as medical imaging, digital cinema, or 3-D imaging, it is worthwhile to investigate how to embed data in JPEG2000 compressed images efficiently. This is the main objective of our research.

We address the problem of combination of informed watermarking and source coding within a single system in order to design a joint JPEG2000 coding and watermarking scheme. We propose to use trellis-coded quantization (TCQ) properties to perform at the same time, quantization of the wavelet coefficients (source coding), and watermarking (channel coding). We chose the TCQ approach because it combines quantization and trellis coding. The other reason is that the TCQ quantization option is provided in part 2 [5] of the JPEG2000 standard.

This paper is organized as follows: We first present the JPEG2000 standard and watermarking methods in the JPEG2000 domain in Section 2. Then, we provide a detailed review of the TCQ quantization in Section 3. We present the proposed joint JPEG2000 coding and watermarking TCQ-based scheme in Section 4. Experimental results are shown in Section 5 to demonstrate the feasibility of the proposed method. Finally, concluding remarks are given in Section 6.

\section{JPEG2000}

\subsection{The JPEG2000 coder}

JPEG2000 [4] is a compression standard developed by the Joint Photographic Experts Group (JPEG). It is based on discrete wavelet transformation, and it provides several important features such as progressive transmission by resolution or quality, better resilience to bit errors, and region of interest (ROI) coding. The main encoding procedures of JPEG2000 (part 1) [4] are illustrated in Fig. 1a. Firstly, the original image undergoes some pre-processing operations (level shifting and color transformation). The image is partitioned into rectangular and non-overlapping tiles of equal size. Each tile is compressed independently using its own set of specified compression parameters. The processed tile is decomposed by the discrete wavelet transform (DWT) into a collection of sub-bands $\left(\mathrm{LL},{ }^{1} \mathrm{HL},{ }^{2} \mathrm{LH}^{3}\right.$, and $\mathrm{HH})^{4}$ which may be organized into increasing resolution levels. The wavelet coefficients are afterward quantized by a dead-zone uniform scalar quantizer. For some applications, ROI coding may be applied. The quantized wavelet coefficients in each sub-band are partitioned into small rectangular blocks which are called code-blocks. Each code-block is encoded independently during the tier 1 encoding stage by using a bit-plane coder called embedded block coding with optimal truncation (EBCOT). So, each coding block has an independent bitstream. These bitstreams are combined into a single bitstream using tier 2 coding based on the result of rate control stage. An efficient rate-distortion algorithm provides possible truncation points of the bitstreams in an optimal way to minimize distortion according to any given target bitrate. The coded data are outputted to the code-stream in packets, and the JPEG2000 file stream is finally formed.

The following steps ensure the decompression of the compressed image as depicted in Fig. 1b: After the tier 2 decoding, the image bitstream is decoded by the EBCOT decoder. Then, the wavelet coefficients are reconstructed during the inverse scalar quantization stage. Afterward, the inverse DWT and the postprocessing operations are performed to reconstruct the image.

\subsection{Watermarking in the JPEG2000 domain}

The JPEG2000 Secured (JPSEC) [7] or part 8 of the standard provides a framework for secure imaging. Watermarking is one of the security tools used in the data integrity service provided by JPSEC. Watermarking is considered here as a post-compression process and is used, along with other cryptographic methods, for image content integrity applications.

Another distinct approach is to insert the watermark during the JPEG2000 compression process. Several watermarking techniques integrated into the JPEG2000 coding scheme have been proposed [8-13]. Few among those methods are quantization-based watermarking schemes combined to JPEG2000 [11-13]. Meerwald [11] developed a watermarking process based on QIM

\footnotetext{
${ }^{1}$ Horizontal and vertical low frequency sub-band.

${ }^{2}$ Horizontal high-frequency and vertical low-frequency sub-band.

${ }^{3}$ Horizontal low-frequency and vertical high-frequency sub-band

${ }^{4}$ Horizontal high-frequency and vertical high-frequency subband.
} 

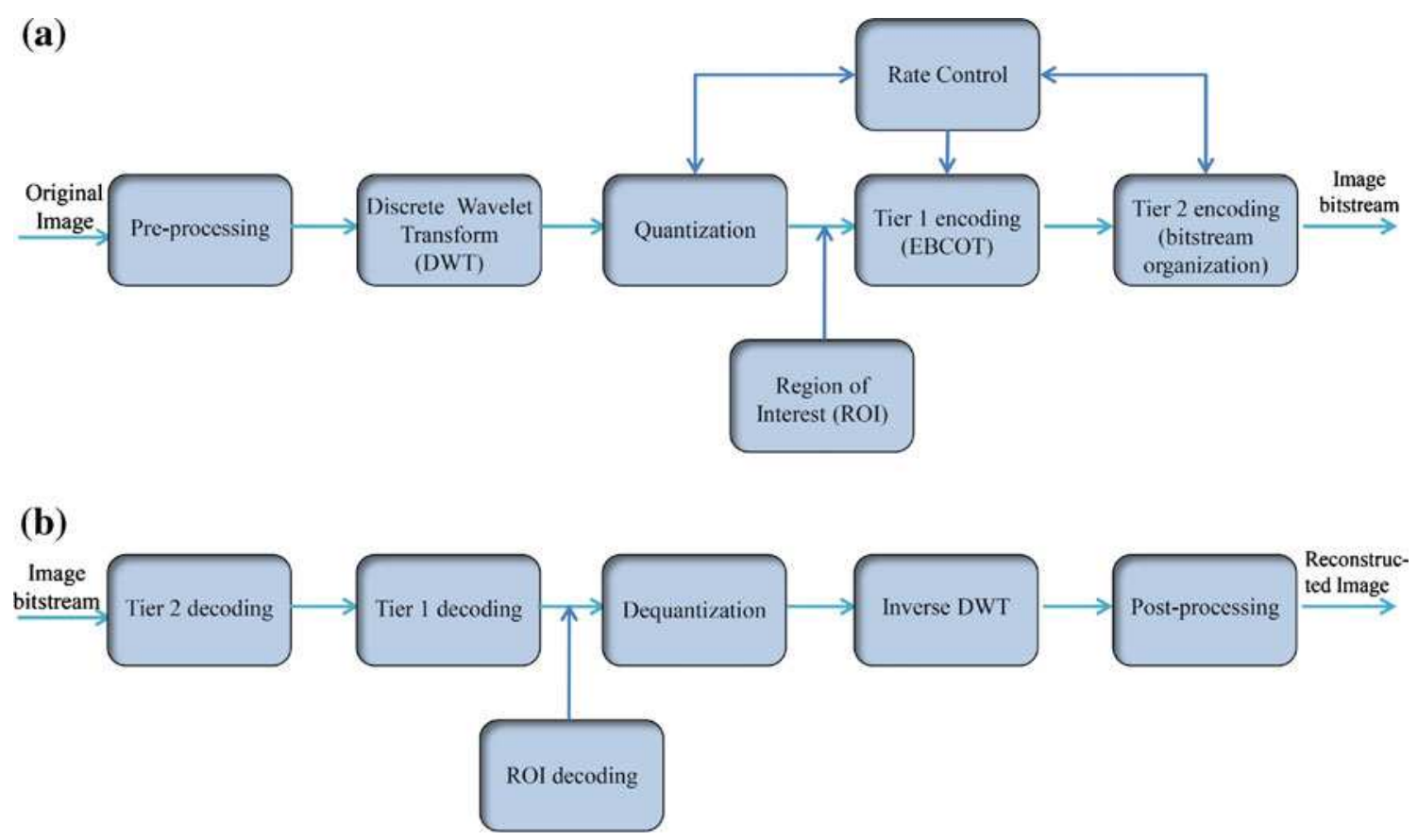

Fig. 1 a Block diagram of the JPEG2000 encoder algorithm. b Block diagram of the JPEG2000 decoder algorithm

integrated into the JPEG2000 coding chain. Despite its robustness, this method does not fulfill the visual quality requirement. $\mathrm{Li}$ and Zhang [10] proposed an adaptive watermarking method integrated in the JPEG2000 coding framework. Wavelet coefficients included in the watermarking process are modified depending on the target bitrate such that the embedded watermark can survive the rate allocation procedure of JPEG2000 without degrading the image quality. Fan and Tsao [8] proposed hiding two kinds of watermarks: a fragile one and a robust one by using a dual pyramid watermarking scheme. The robust pyramid watermark is designed to conceal secret information inside the image so as to attest to the origin of the host image. The fragile pyramid watermark is designed to detect any modification of the host image. Schlauweg et al. [12] have developed a semi-fragile authentication watermarking scheme by using an extended scalar quantization and hashing scheme in the JPEG2000 coding pipeline. This authentication scheme is secure, but the embedding of the watermark induces poor quality performances. Fan et al. [9] proposed ROI-based watermarking scheme. The embedded watermark can survive ROI processing, progressive transmission, and rate-distortion optimization. The only drawback of this method is that it works only when the ROI coding functionality of JPEG2000 is activated. Ouled-Zaid et al. [13] have proposed to integrate a modified QIM scheme in JPEG2000 part 2.
The QIM modification consists of reducing the distortion caused during quantization-based watermarking by using a non-linear scaling.

We can notice that two watermarking approaches have been commonly used in the state-of-the-art. One is to embed data into the wavelet coefficients before the quantization stage [13]. The other is to operate directly on the quantization indices after the quantization process [8-12]. In contrast of this conventional approaches, our proposed method embeds the watermark during the quantization process of JPEG2000.

\section{Trellis-coded quantization in JPEG2000}

Several quantization options are provided within JPEG2000. Part 2 [5] allows for the use of TCQ as a replacement for scalar quantization. TCQ is a particular kind of vector quantization proposed by Marcellin and Fischer [14]. The variant of TCQ used in the part 2 of the JPEG2000 standard is the entropy-coded TCQ [22]. A uniform scalar quantizer with step size $\Delta$ is partitioned into four subsets called $D_{0}, D_{1}, D_{2}$, and $D_{3}$. Subsets $D_{j}, j=0,1,2,3$ are used to label the branches of a trellis as shown in Fig. 2. The two scalar quantizers associated with each state in the trellis are combined into union quantizers $A_{0}$ and $A_{1}$. The two union quantizers $A_{0}=D_{0} \cup D_{2}, A_{1}=D_{1} \cup D_{3}$ are illustrated in 
Fig. 2 A single stage of an eight-state trellis with branch labeling used in JPEG2000

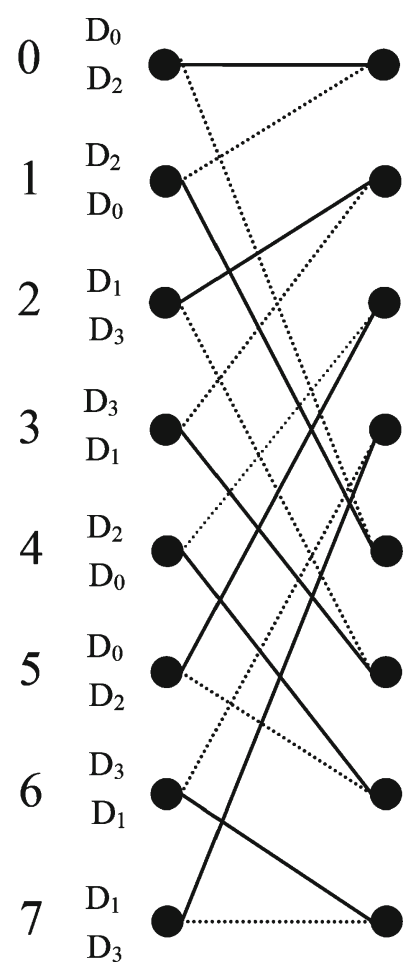

\section{The proposed joint JPEG2000 and watermarking scheme}

Fig. 3. This figure shows the reconstruction values $\hat{x}$ corresponding to each union quantizer along with the corresponding union quantizer indices $q\left(A_{i}\right)$. Notable features of this figure are that the codebook is uniform with step size $\Delta$ and that the zero codeword appears in two subsets, $D_{0}$ and $D_{1}$.

At each state in the trellis, we can choose between one of the two quantizers belonging to the union quantizer to quantize the input sequence $\mathbf{x}$. Quantization proceeds via the Viterbi algorithm [15] to determine the trellis path that minimizes the mean-squared error between the input sequence and output codewords. The Viterbi Algorithm produces two sequences: The first one is a binary sequence defining the minimum distortion path. The second one is the sequence of corresponding quantization indices. Note that by construction of the trellis, the least significant bit of each union
TCQ have already been used in data hiding [18-21] and watermarking $[16,17]$. These methods rely on the following principle: The paths in the trellis are forced by the values of the message, and the samples of the host signal are quantized with the subset corresponding to the trellis path. We propose another TCQ-based technique which is independent of the path. The proposed joint system allows both quantization of wavelet coefficients and watermark embedding without integrating an additional stage for watermarking in the JPEG2000 coding/decoding chain.

\subsection{Overview of the proposed joint scheme}

quantizer index $q\left(A_{j}\right)$ corresponds to the path since are two possible codewords for each index $\left(D_{0}\right.$ or the path through the trellis. Given the initial state and by construction of the trellis, the TCQ indices from union quantizers $A_{0}$ and $A_{1}$ provide all information necessary to reconstruct the wavelet coefficients.

The block diagram of the joint JPEG2000 part 2 and watermark embedding scheme is illustrated in Fig. 4. The classical TCQ quantization component of the JPEG2000 encoder and decoder is replaced by a hybrid TCQ module which can perform at the same time quantization and watermark embedding. The quantization algorithm employs a modified version of the TCQ described in Section 3. One of the most important parameter to consider is the selection of the wavelet coefficients that must be included in the watermarking process. According to the wavelet decomposition, the LL sub-band carries out the low frequencies which represents the most significant data of the transformed image. In order to avoid considerable quality degradation in the reconstructed image, the wavelet coefficients
Fig. 3 Union quantizers $A_{0}$ and $A_{1}$ for TCQ in JPEG2000. $\hat{x}$ is the reconstruction value and $q\left(A_{i}\right)$ is the union quantizer index corresponding to this reconstruction value

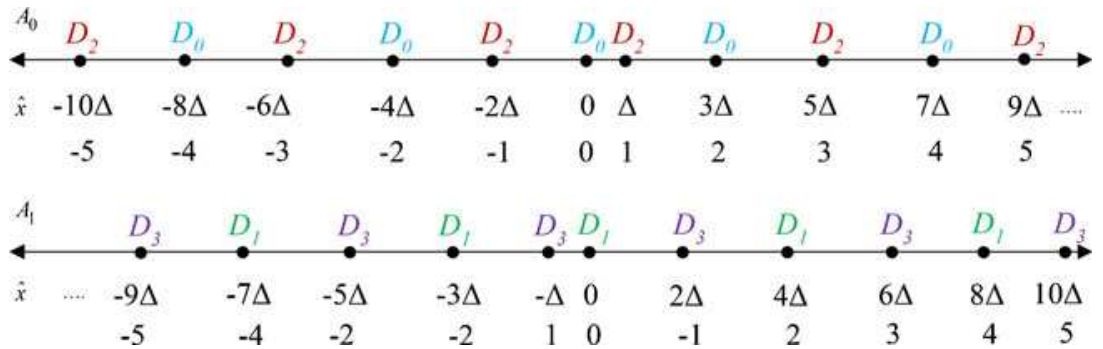




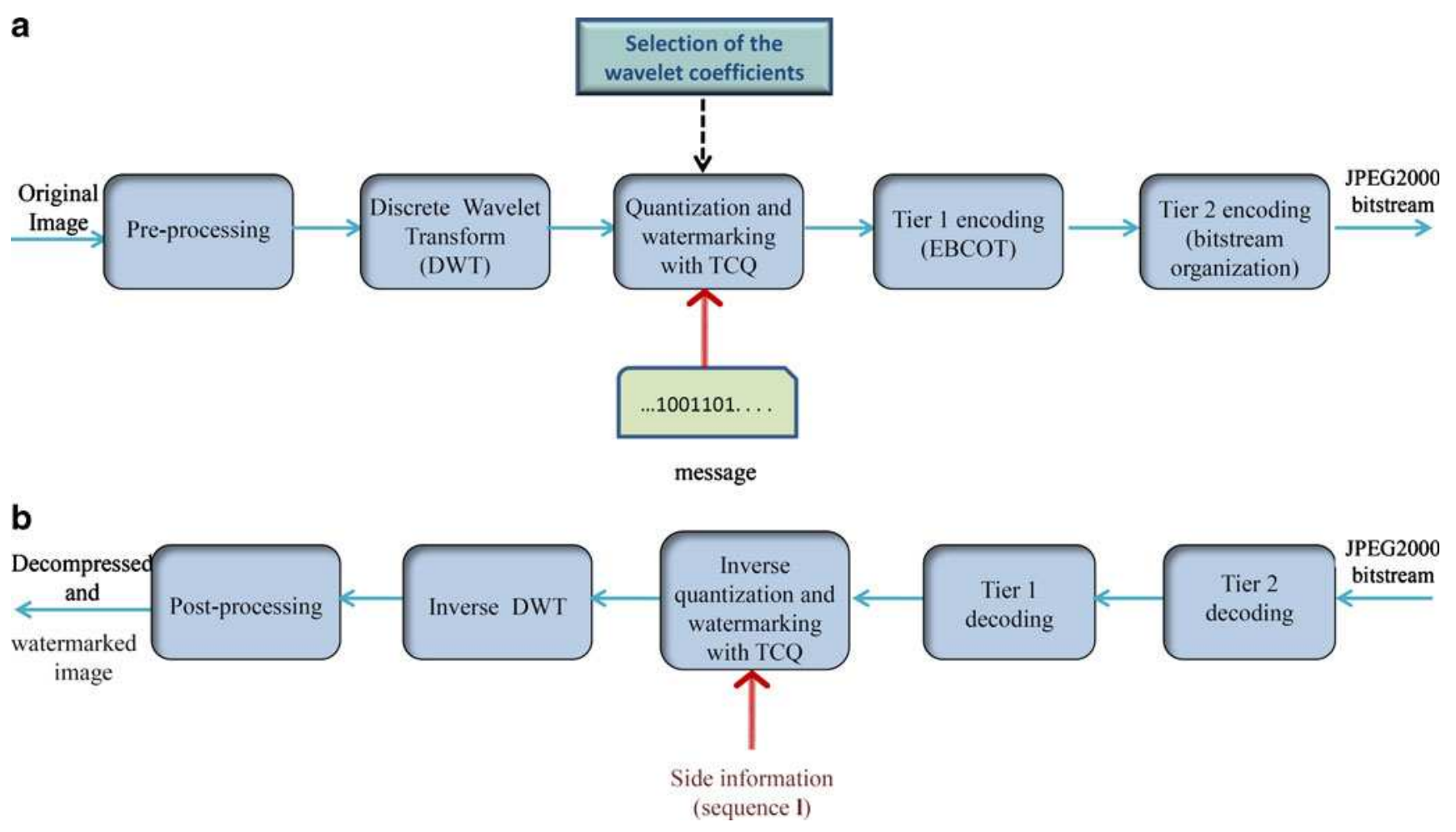

Fig. 4 The joint JPEG2000/watermarking scheme: a compression process, $\mathbf{b}$ decompression process

of this sub-band are not selected by the watermarking process. Therefore, we choose to embed the watermark in the HL, LH, and HH detail sub-bands of selected resolution levels. Wavelet coefficients of the other subbands are quantized with the classical TCQ algorithm of JPEG2000 part 2. The watermarking payload is determined by the number of detail sub-bands included in the watermarking process. The payload increases when we add more detail sub-bands from a new selection of resolution levels.

\subsection{Description of the TCQ-based watermarking method}

We aim to design a watermarking algorithm to quantize and watermark wavelet coefficients at the same time by using a TCQ-based quantization module. Our method is based on the principles of the QIM [1] approach associated with a trellis. We replace the uniform scalar quantizers used in JPEG2000 part 2 by shifted scalar quantizers with the same step size $\Delta$ as for the original ones. We can also use a higher step size by multiplying the original step size by a constant. These quantizers differ from the previous quantizers by the introduction of a shift $d$ which is randomly obtained with a uniform distribution over $[-\Delta / 2, \Delta / 2] .{ }^{5}$ We propose the following principle: If the bit to embed is the bit 0 , then the quantizer $D_{j}^{0}, j=0,1,2,3$ with the shift $d_{0}$ is used. If it is the bit 1 , then we employ the quantizer $D_{j}^{1}$ with the shift $d_{1}$ satisfying the condition: $\left|d_{0}-d_{1}\right|=\Delta / 2$.

For each transition $i$ in the trellis, two shifts $\mathbf{d}_{0}[i]$ and $\mathbf{d}_{1}[i]$ and four union quantizers $A_{0, i}^{0}=D_{0, i}^{0} \cup$ $D_{2, i}^{0}, A_{1, i}^{0}=D_{1, i}^{0} \cup D_{3, i}^{0}, A_{0, i}^{1}=D_{0, i}^{1} \cup D_{2, i}^{1}, A_{1, i}^{1}=$ $D_{1, i}^{1} \cup D_{3, i}^{1}$ are constructed. Thus, we will have two groups of union quantizers for the trellis structure used in our approach: the group 0, which consists of all shifted union quantizers corresponding to the watermark embedded bit 0 , and the group 1 , which incorporates shifted union quantizers corresponding to the embedded bit 1 . Two dithered vectors $\mathbf{d}_{0}$ and $\mathbf{d}_{1}$ are constructed: Group 0 is associated to $\mathbf{d}_{0}$ and group 1 is associated to $\mathbf{d}_{1}$. The trellis structure used in the proposed method has four branches leaving each state

\footnotetext{
${ }^{5}$ Shuchman [23] showed that the subtractive dithered quantization error does not depend on the quantizer input when the dither signal d has a uniform distribution within the range of one quantization bin $(d \in[-\Delta / 2, \Delta / 2])$ leading to an expected squared error of $E^{2}=\Delta^{2} / 12$.
} 
(Figs. 5 and 6a). For each state, two union quantizers instead of one are associated with branches exiting this state.

Let us consider a binary message $\mathbf{m}$ to embed and a host signal $\mathbf{x}$. The embedding function $\operatorname{Emb}(\mathbf{x}, \mathbf{m})=\hat{\mathbf{x}}$ incorporates the message $\mathbf{m}$ into $\mathbf{x}$, yielding the watermarked signal $\hat{\mathbf{x}}$. For a given transition $i, \hat{\mathbf{x}}[i]$ is obtained by quantization of the host component $\mathbf{x}[i]$ and Emb can be expressed as follows:

$\operatorname{Emb}(\mathbf{x}[i], \mathbf{m}[i])=\left(\left\lfloor\frac{\left|\mathbf{x}[i]-\mathbf{d}_{\mathbf{m}[i]}\right|}{\Delta}\right\rfloor\right) \times \Delta+\mathbf{d}_{\mathbf{m}[i]}$,

where $\mathbf{d}_{\mathbf{m}}$ i] is the shift introduced in the selected quantizer of step size $\Delta$. The watermark embedding process is split into two steps to perform watermarking within JPEG2000. The first step is achieved during the quantization stage of the JPEG2000 compression process. The quantization stage produces the sequence of TCQ quantization indices q. For each transition $i$ in the trellis, the union quantizers are selected according to the value $\mathbf{m}[\mathrm{i}]$. The trellis is thus modified in order to remove all the branches that are not labeled with the

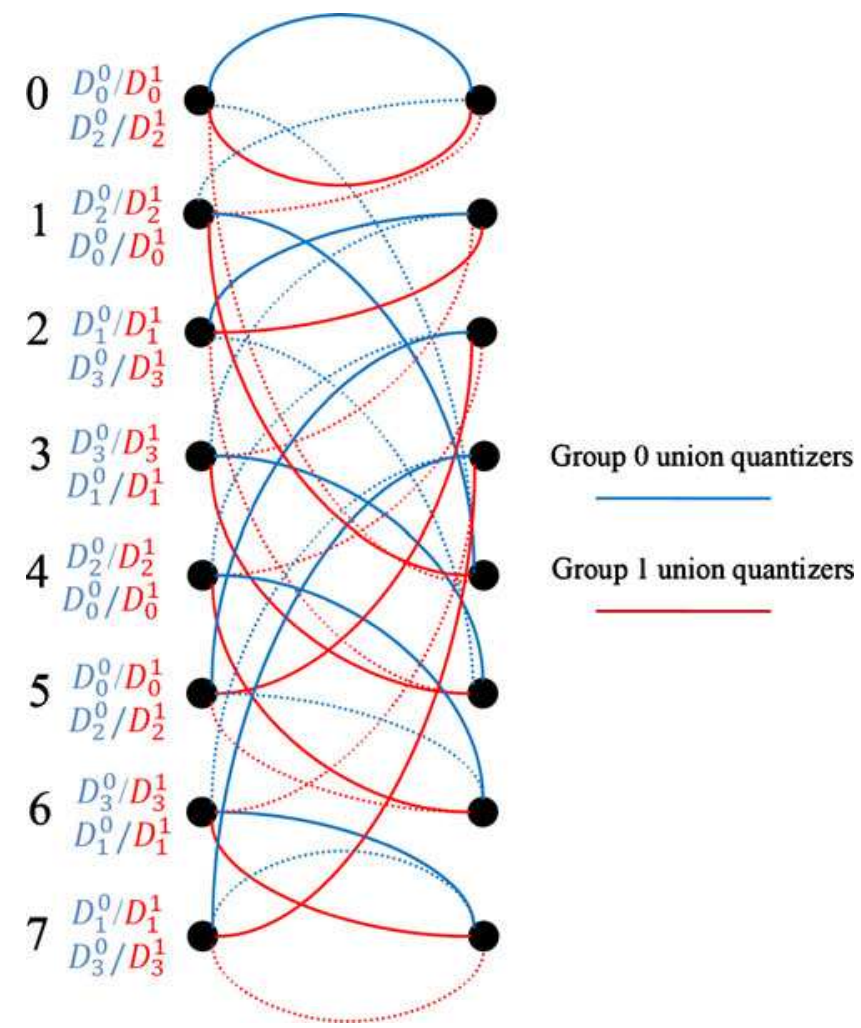

Fig. 5 A single stage of the trellis structure used our joint watermarking/JPEG2000 scheme with branch labeling union quantizers that encode the message as illustrated in Fig. 6b. The subsets $D_{j, i}^{\mathbf{m}[\mathbf{i}]}, j=0,1,2,3$ are associated to the branches of the modified trellis. The quantization index $\mathbf{q}[\mathrm{i}]$ is given by:

$\mathbf{q}[i]=Q_{D_{j . i}^{\mathbf{m}[i]}}(\mathbf{x}[i])$,

where $Q$ is the quantization function of JPEG2000, $\mathbf{m}[\mathrm{i}]$ is the bit to embed at transition $i$, and $D_{j, i}^{\mathbf{m}[\mathbf{i}]}$ is the shifted quantizer. For a given step size $\Delta, \mathbf{q}[\mathbf{i}]$ can be computed as:

$\mathbf{q}[i]=\operatorname{sign}\left(\mathbf{x}[i]-\mathbf{d}_{\mathbf{m}[i]}[i]\right)\left\lfloor\frac{\left|\mathbf{x}[i]-\mathbf{d}_{\mathbf{m}[i]}[i]\right|}{\Delta}\right\rfloor$,

where $\mathbf{d}_{\mathbf{m}[i]}[i]$ is the shifting of the shifted quantizer $D_{j, i}^{\mathbf{m}[i]}$.

The second step is performed during the inverse quantization stage of the JPEG2000 decompression process. The trellis must be pruned in order to obtain the same trellis employed during the first step of the watermarking process. In addition to $\mathbf{q}$, the sequence $\mathbf{I}$ is necessary to retrieve the modified trellis structure that have been used during the quantization stage. The reconstructed values $\hat{\mathbf{x}}$ are produced as:

$\hat{\mathbf{x}}[i]=\bar{Q}_{D_{j, i}^{\mathbf{m}[i]}}^{-1}(\mathbf{q}[i])$,

where $\bar{Q}^{-1}$ is the inverse quantization function of JPEG2000. For a given step size $\Delta$, the reconstructed value $\hat{\mathbf{x}}$ can be computed as:

$\hat{\mathbf{x}}[i]=\operatorname{sign}(\mathbf{q}[i])(|\mathbf{q}[i]|+\delta) \Delta+\mathbf{d}_{\mathbf{m}[i]}[i]$,

where $\delta$ is a user selectable parameter within the range $0<\delta<1$ (typically $\delta=0.5$ ).

The proposed watermarking method have similarities with the dirty paper trellis codes (DPTC) [3]. Both methods rely on the use of a modified trellis associated to a codebook. However, we use a quantization codebook partitioned into subsets while Miller et al. use a pseudo-random code [3]. Moreover, the embedding of the watermark is done in a different way. Our joint scheme integrates a quantization-based method instead of DPTC codes, which optimally embed a watermark by applying an iterative embedding procedure with the constraint of minimizing the perceptual distance and maintaining constant robustness. The codeword is determined by using a correlation instead of a quantization.

\subsection{Watermark embedding}

The watermark embedding process is performed independently into each code-block. In order to add more 


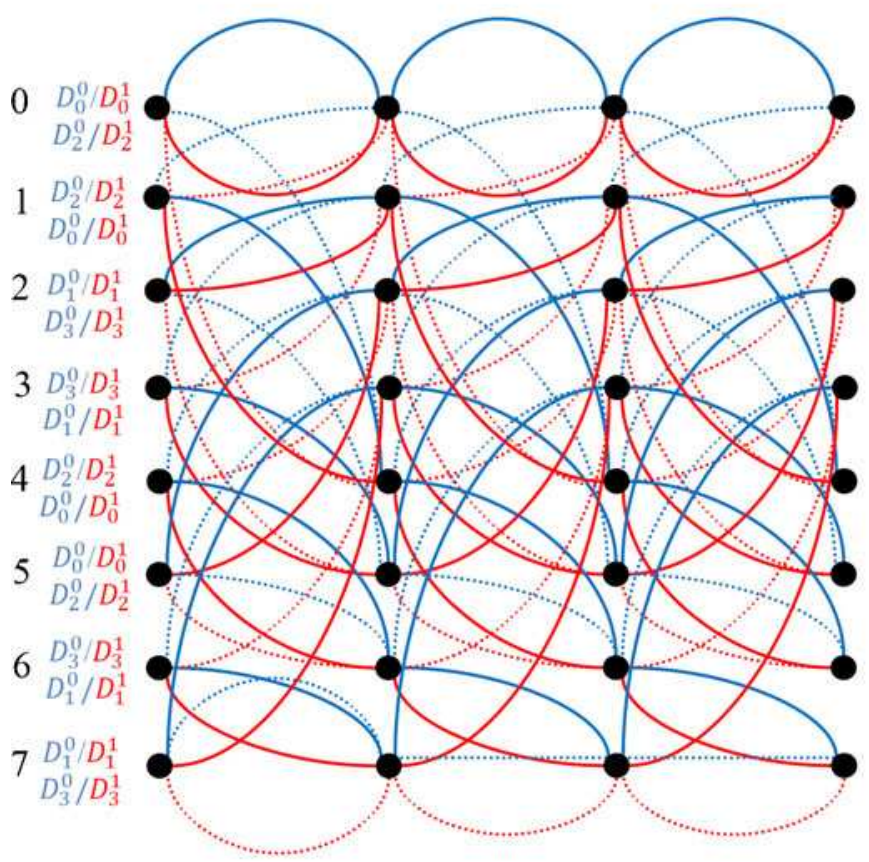

(a)

Fig. 6 a A three-stage modified trellis structure, $\mathbf{b}$ insertion of the message $m=\{1,0,1\}$ : All the branches that are not labeled with the union quantizers that encode the message are removed.

robustness to the message, we encode it with an error correcting code. After that, we shuffle (scatter) pseudorandomly the bits of the coded message with a secret key.

Quantization For each code-block $\mathbf{C}$, the quantization/watermark embedding procedures are:

- Computation of the shiftings $\mathbf{d}_{0}$ and $\mathbf{d}_{1}$ : We use a pseudo-random generator initialized by the secret key $k$ to compute the shiftings.

- Generation of the group 0 and group 1 union quantizers: For each transition $i$, we design shifted scalar quantizers. We label the branches of the trellis with these quantizers. Figure $6 \mathrm{a}$ shows a threestage trellis structure used in our joint scheme. All the branches in the trellis are also labeled with reference numbers. At each transition, the straight branches are referenced by the value 0 and the dotted branches by value 1 .

- Trellis pruning: The trellis is simplified so that all the branches through the trellis, and thus all the associated union quantizers encode the message $\mathbf{m}$ as illustrated in Fig. 6b. For each transition, we save

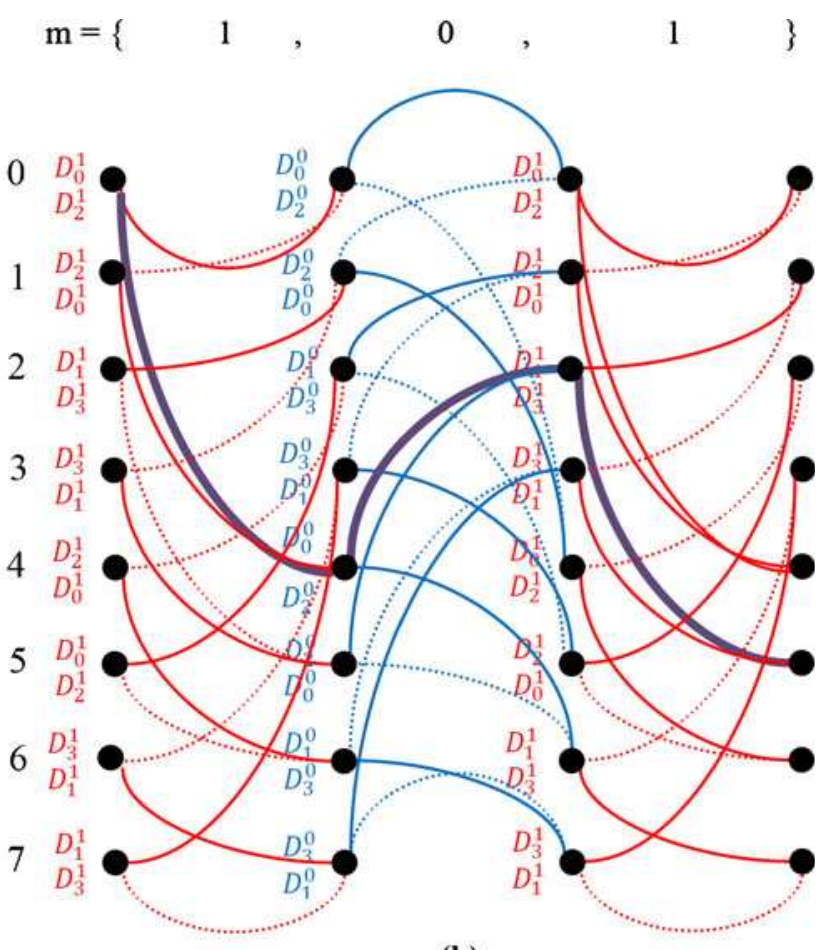

(b)

The bold branches represent the optimal path calculated by the Viterbi algorithm

the reference number of the surviving branches. We obtain the sequence $\mathbf{l}_{C}$.

- Finding the optimal path: The initial state of the given trellis structure is set to 0 . The Viterbi Algorithm [15] is applied in order to find the minimum distortion path (Fig. 6b). The TCQ indices are produced (Eq. 3).

The sequences $\mathbf{I}_{C}$ are combined to form the sequence I. The obtained sequence $\mathbf{I}$ is afterward crypted and stored in a file which is transmitted to the joint decoder as side information.

Inverse quantization The watermark embedding is completed during the inverse quantization of the JPEG2000 decompression stage. The image bitstream is decoded by the EBCOT decoder (tier 2 and tier 1 decoding) to obtain the sequence of decoded TCQ indices. For each code-block $\mathbf{C}$, the inverse quantization steps are the following:

- Computation of the shiftings $\mathbf{d}_{0}$ and $\mathbf{d}_{1}$

- Generation of the group 0 and group 1 union quantizers 
- Retrieval of the trellis structure used during the quantization stage: The trellis structure with four branches leaving each state is generated. Each branch of the trellis is afterward labeled with the shifted quantizers and with the reference numbers. The sequence I enables us to retrieve the pruned trellis used during the quantization stage. For each transition $i$ in the trellis, the pruning is done by removing the branches that have their reference number not equal to $\mathbf{l}_{C}[i]$

- Inverse quantization: The pruned trellis is used to reconstruct the wavelet coefficients. Given the TCQ indices, the embedding of the watermark is achieved during the computation of the reconstructed wavelet coefficients (Eq. 5).

\subsection{Watermark extraction}

The watermark recovery from the watermarked/decompressed image is a blind watermarking extraction process. The following operations are performed:

- Apply the DWT: we apply the DWT on the decompressed watermarked image. Each sub-band included in the watermarking process is partitioned into blocks of same size as the JPEG2000 codeblocks. The coefficients belonging to the current block are stored in the vector $\mathbf{y}$. The following steps are repeated for each processed block.

- Retrieve the shiftings $\mathbf{d}_{0}$ and $\mathbf{d}_{1}$ : We retrieve the shiftings by using the secret key $k$, and we set the union quantizers group 0 and group 1 .

- Perform the TCQ quantization: The decoder applies the Viterbi algorithm to the entire trellis (Fig. 6a). This identifies the path that yields the minimum quantization distortion between $\mathbf{y}$ and the output codewords. The coded message is then recovered by looking at the TCQ codebook labeling represented by the branches in that path. After that, we invert the schuffle and apply the decoding of the error correcting code to retrieve the message.

\subsection{Discussion on the security of the proposed watermarking method}

In this section, the security of the proposed joint scheme is discussed. The security of a watermarking system concerns its capability to resist to intentional attacks. All the parameters of the watermarking scheme are assumed to be public except the secret key used for embedding. The objective of an intentional attacker is to estimate the secret parameters used during embed- ding. An accurate estimate of the secret key allows to implement a number of different attacks such as message modification, message copy, or message erasure while keeping a very low distortion. Different classes of security attacks can be considered [25]. We place this study under the general watermark only attack (WOA) scenario where the attacker has only access to several watermarked contents. He knows the step size values of the JPEG2000 TCQ quantizers, the trellis structure, and the repetition code rate whereas he ignore the selected resolution levels (the selected wavelet coefficients), the embedded message, the shuffle parameters, and the secret key needed to compute the dither vectors. He has access to a collection of images watermarked with the same secret key. These images are called observations.

The security of the proposed watermarking scheme relies on the dither signal and the secret pseudorandom seed used for shuffling the bits of the coded message (secret permutation). The TCQ codebooks are randomized by means of a pseudo-random dither signal. The dither vectors $\mathbf{d}_{0}$ and $\mathbf{d}_{1}$ introduce a secret shift in the TCQ quantizers. If the same dither signal is reused, the observation of several watermarked images can provide sufficient information for an attacker to estimate the dither signal. This can be done by exploiting the information leakage between several watermarked contents by means of information theoretic measures such as equivocation or mutual information between the dither signal and the observations [24]. The use of a trellis adds more complexity to the estimation problem because the attacker has to estimate all the components of the dither vectors in one time by testing all possible trellis paths [16]. It is thus more difficult for the attacker to estimate the secret key, and he needs to have a large amount of observations to be able to entirely estimate the dither signal. Once the attacker has accurately estimate the dither vectors, he can obtain the shuffled coded message. He cannot obtain the embedded message because he does not have the secret key used for the shuffle. However, it is still possible for the attacker to invert the shuffle. In this case, the problem of inverting the shuffle relies on the security of the shuffle itself. So, the shuffle of the coded message adds more security to the watermarking method by keeping the embedded message hard to read for an unauthorized user.

The proposed watermarking scheme is vulnerable to security attacks in the WOA scenario as it is the case for quantization watermarking schemes [24]. The use of a trellis and the shuffle of the embedded message allows to enhance the security level of the proposed scheme. It is also possible to increase the security of 
the watermarking system by proceeding to the shuffle of the wavelet coefficients of the selected code-blocks before embedding.

\section{Experimental results}

To implement our joint JPEG2000 and watermarking scheme, we chose to use the OpenJPEG library ${ }^{6}$ which is a JPEG2000 part 1 open-source codec written in C language. We replaced the scalar uniform quantization component by a JPEG2000 part 2 compliant TCQ quantization module. The following set of compression and watermark parameters were fixed: irreversible DWT 9-7, five levels of wavelet decomposition, one tile, no ROI coding, size of code-block: $64 \times 64$ for the second and third levels of resolution, $32 \times 32$ for the fourth level, and $16 \times 16$ for the fifth level. A message of 1,020 bits length is inserted in the detail subbands of the second to the fifth resolution level. The joint scheme embed 1 bit of the (non-coded) message for every 257 pixels in an image of size $512 \times 512$. The message is encoded with a very simple repetition code of 1/64 rate. The choice of using this low rate is dictated by the fact that the higher frequency sub-bands have low energy. $\Delta_{\mathrm{TCQ}}$ is the step size of the shifted TCQ quantizers. We use different values of $\Delta_{\mathrm{TCQ}}$ in our experiments: $\Delta / 4, \Delta / 2, \Delta$, and $2 \Delta$ where $\Delta$ is the JPEG2000 original step size value used in JPEG2000 part $1 . \Delta / 4$ is the TCQ quantizer step size value used in JPEG2000 part 2.

The evaluation of the performances of the proposed joint scheme covers two aspects: On one hand, the compression performances are studied. On the other hand, watermarking performances are investigated. Therefore, we use three kinds of experimental protocols: The first one studies the compression performances of the proposed joint scheme under various compression bitrates. The second one examines the impact of JPEG2000 compression on the watermark and the compression rate/imperceptibility trade-off. The last one studies the robustness of watermarked images against four attacks.

\subsection{First experimental protocol: compression performances}

Series of experiments on different JPEG2000 grayscale test images of size $512 \times 512$ have been performed

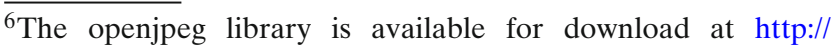
www.openjpeg.org.
}

to evaluate the compression performances of the proposed joint scheme. We set the compression ratio from 2.5 to 0.2 bpp. Quality assessment was carried out using two objective evaluation criteria, pick signal-to-noise ratio (PSNR) and structural similarity (SSIM) ${ }^{7}$

Table 1 shows the PSNR and the SSIM results obtained for four well-known test images: Bike, Clown, Lena, and Peppers with $\Delta_{\mathrm{TCQ}}=\Delta / 4$ (the TCQ quantizer step size value used in JPEG2000 part 2). We notice that the joint scheme exhibits very good quality performances in terms of PSNR. For all the tested bitrates, the obtained values are greater than $30 \mathrm{~dB}$ except for Peppers image at $0.2 \mathrm{bpp}(29.22 \mathrm{~dB})$. The SSIM values are good at high bitrates and prove that our joint scheme provides good perceptual quality. We note that the quality degradation resulting from watermark embedding is very small when we compare, respectively, between the PSNR (and the SSIM) computed for the JPEG2000 compressed image and those computed for the compressed and watermarked image. Moreover, the PSNR obtained at some bitrates are slightly greater than those obtained with the classical JPEG2000 part 2 coder. This is due to the use of shifted TCQ quantizers during inverse quantization of the decoded wavelet coefficients. This sometimes provides reconstructed values closer to the original ones than those obtained with the JPEG2000 TCQ quantizers. It means that, compared to JPEG2000 compression, the visual quality obtained with our joint scheme is similar and sometimes better than with the conventional JPEG2000 coder. An example of watermarked and compressed image at different bitrates is presented in Fig. 7 for the Lena image.

The bitstream produced by the proposed joint scheme is compatible with the JPEG2000 part 2 image coding system. Conventional JPEG2000 part 2 decoders can therefore decode the watermarked bitstream and produce a decompressed image. In this case, the two union quantizers $A_{0}$ and $A_{1}$ are used to dequantize the decoded wavelet coefficients instead of group 0 and 1 dithered union quantizers. However, the JPEG2000 decoder produces an image which is close in quality to the one decoded with our joint scheme as shown in Table 2. The PSNR and SSIM results are similar and sometimes better than those obtained with the joint decoder $\left(\Delta_{\mathrm{TCQ}}=\Delta / 4\right)$.

\footnotetext{
${ }^{7}$ SSIM is a perceptual measure exploiting human visual system properties. The SSIM values are real positive numbers lower or equal to 1 . Stronger is the degradation and lower is the SSIM measure.
} 
Table 1 PSNR (decibel) and SSIM for compressed image tests obtained with the joint scheme and comparison with the conventional JPEG2000 part $2 \operatorname{coder}\left(\Delta_{\mathrm{TCQ}}=\Delta / 4\right)$

\begin{tabular}{|c|c|c|c|c|c|}
\hline \multirow[t]{2}{*}{ Image test } & \multirow[t]{2}{*}{ bitrate (bpp) } & \multicolumn{2}{|l|}{ PSNR (dB) } & \multicolumn{2}{|l|}{ SSIM } \\
\hline & & JPEG2000 & Our joint scheme & JPEG2000 & Our joint scheme \\
\hline \multirow[t]{6}{*}{ Bike } & 2.5 & 43.23 & 42.99 & 0.9775 & 0.9785 \\
\hline & 2 & 39.64 & 39.66 & 0.9547 & 0.9546 \\
\hline & 1.6 & 39.33 & 39.40 & 0.9343 & 0.9342 \\
\hline & 1 & 38.11 & 38.17 & 0.8852 & 0.8853 \\
\hline & 0.5 & 36.51 & 36.49 & 0.8031 & 0.8017 \\
\hline & 0.2 & 33.52 & 33.50 & 0.6737 & 0.6814 \\
\hline \multirow[t]{6}{*}{ Clown } & 2.5 & 44.08 & 44.70 & 0.9885 & 0.9886 \\
\hline & 2 & 42.78 & 42.83 & 0.9817 & 0.9815 \\
\hline & 1.6 & 40.77 & 41.04 & 0.9741 & 0.9740 \\
\hline & 1 & 38.71 & 38.93 & 0.9596 & 0.9593 \\
\hline & 0.5 & 35.76 & 35.85 & 0.9249 & 0.9247 \\
\hline & 0.2 & 31.09 & 31.20 & 0.8384 & 0.8393 \\
\hline \multirow[t]{6}{*}{ Lena } & 2.5 & 47.47 & 47.55 & 0.9836 & 0.9834 \\
\hline & 2 & 45.33 & 45.27 & 0.9749 & 0.9749 \\
\hline & 1.6 & 43.38 & 43.09 & 0.9661 & 0.9658 \\
\hline & 1 & 41.55 & 41.64 & 0.9490 & 0.9491 \\
\hline & 0.5 & 40.03 & 39.90 & 0.9226 & 0.9227 \\
\hline & 0.2 & 36.56 & 36.55 & 0.8700 & 0.8711 \\
\hline \multirow[t]{6}{*}{ Peppers } & 2.5 & 43.13 & 43.43 & 0.9800 & 0.9802 \\
\hline & 2 & 39.69 & 39.75 & 0.9649 & 0.9651 \\
\hline & 1.6 & 39.20 & 39.16 & 0.9531 & 0.9528 \\
\hline & 1 & 39.03 & 39.08 & 0.9273 & 0.9273 \\
\hline & 0.5 & 36.50 & 36.53 & 0.8878 & 0.8875 \\
\hline & 0.2 & 29.35 & 29.22 & 0.8378 & 0.8376 \\
\hline
\end{tabular}

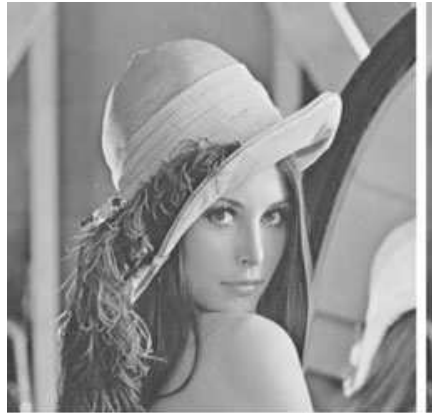

(a)

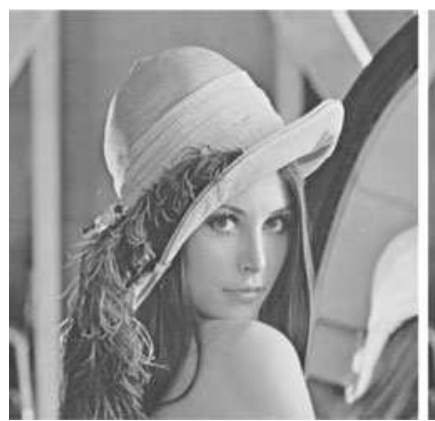

(c)

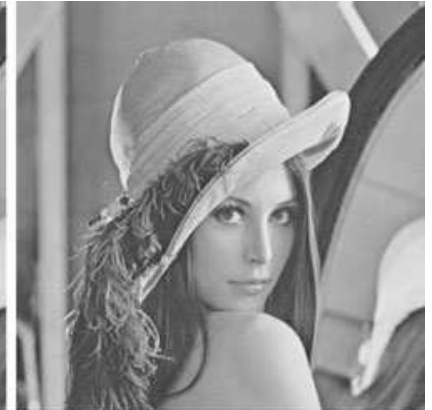

(b)

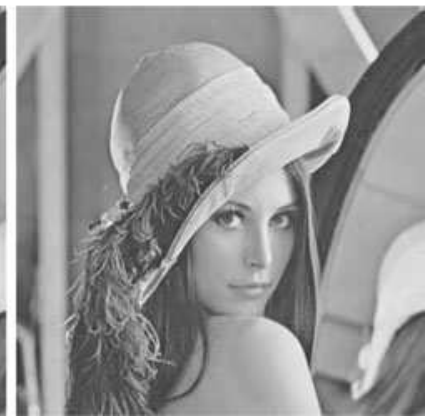

(d)
Fig. 7 Lena image watermarked and compressed with our joint scheme at different bitrates $\left(\Delta_{\mathrm{TCQ}}=\Delta / 4\right)$ : a original image, b 2 bpp, c 1 bpp, d 0.2 bpp
5.2 Second experimental protocol: impact of the compression process on the watermarking performances

We propose to study the impact of JPEG2000 compression on the watermark extraction. We have performed our experiments on 200 grayscale images of size $512 \times 512 .{ }^{8}$ We compute the average percentage of correct emdedded bits extracted at different bitrates. We note that the embedded watermark information is completely recovered for all compression bitrates for $\Delta / 2, \Delta$, and $2 \Delta$. For $\Delta / 4$, the percentage of correct extraction of the embedded watermark is equal to $99 \%$ at 2.5 and $2 \mathrm{bpp}, 98 \%$ at $1.6,1$, and $0.5 \mathrm{bpp}$, and $96 \%$ at $0.2 \mathrm{bpp}$.

Figures 8 and 9, respectively, show the average PSNR and the average SSIM curves at different bitrates and different step size values for the considered image database. We should notice that there is a trade off between the quantizer step size needed for a correct extraction of the watermark and the expected quality of the decompressed/watermarked image. The step size

\footnotetext{
${ }^{8}$ These images are from the BOWS2 database which is located at http://bows2.gipsa-lab.inpg.fr.
} 
Table 2 Comparison between the PSNR (decibel) and SSIM of the images obtained from the watermarked bitstream with the proposed joint JPEG2000/watermarking decoder and the JPEG2000 part 2 decoder $\left(\Delta_{\mathrm{TCQ}}=\Delta / 4\right)$

\begin{tabular}{|c|c|c|c|c|c|}
\hline \multirow[t]{2}{*}{ Image test } & \multirow[t]{2}{*}{ bitrate (bpp) } & \multicolumn{2}{|l|}{ PSNR (dB) } & \multicolumn{2}{|l|}{ SSIM } \\
\hline & & Joint decoder & JPEG2000 decoder & Joint decoder & JPEG2000 decoder \\
\hline \multirow[t]{6}{*}{ Bike } & 2.5 & 42.99 & 43.00 & 0.9785 & 0.9778 \\
\hline & 2 & 39.66 & 39.67 & 0.9546 & 0.9546 \\
\hline & 1.6 & 39.40 & 39.39 & 0.9342 & 0.9342 \\
\hline & 1 & 38.17 & 38.22 & 0.8853 & 0.8853 \\
\hline & 0.5 & 36.49 & 36.47 & 0.8017 & 0.8018 \\
\hline & 0.2 & 33.50 & 33.48 & 0.6737 & 0.6815 \\
\hline \multirow[t]{6}{*}{ Clown } & 2.5 & 44.70 & 44.57 & 0.9886 & 0.9885 \\
\hline & 2 & 42.83 & 42.71 & 0.9815 & 0.9814 \\
\hline & 1.6 & 41.04 & 40.99 & 0.9740 & 0.9739 \\
\hline & 1 & 38.71 & 38.89 & 0.9593 & 0.9593 \\
\hline & 0.5 & 35.85 & 35.83 & 0.9247 & 0.9248 \\
\hline & 0.2 & 31.20 & 31.19 & 0.8393 & 0.8393 \\
\hline \multirow[t]{6}{*}{ Lena } & 2.5 & 47.55 & 47.36 & 0.9834 & 0.9833 \\
\hline & 2 & 45.27 & 45.19 & 0.9749 & 0.9748 \\
\hline & 1.6 & 43.09 & 43.24 & 0.9658 & 0.9657 \\
\hline & 1 & 41.64 & 41.70 & 0.9491 & 0.9491 \\
\hline & 0.5 & 39.90 & 39.89 & 0.9227 & 0.9228 \\
\hline & 0.2 & 36.55 & 36.49 & 0.8711 & 0.8712 \\
\hline \multirow[t]{6}{*}{ Peppers } & 2.5 & 43.43 & 43.26 & 0.9802 & 0.9800 \\
\hline & 2 & 39.75 & 39.69 & 0.9651 & 0.9649 \\
\hline & 1.6 & 39.16 & 39.12 & 0.9528 & 0.9528 \\
\hline & 1 & 39.08 & 39.07 & 0.9273 & 0.9273 \\
\hline & 0.5 & 36.53 & 36.52 & 0.8875 & 0.8877 \\
\hline & 0.2 & 29.22 & 29.23 & 0.8376 & 0.8377 \\
\hline
\end{tabular}

used in JPEG2000 is small. It is big enough to ensure a correct extraction of the watermark. Nevertheless, the watermarks will not survive in case of strong power attacks. When using a larger $\Delta_{\mathrm{TCQ}}$, the fidelity is deteriorated because the distance between the quantization points grows. However, the advantage of using a larger $\Delta_{\mathrm{TCQ}}$ value is that an improved robustness is obtained. The PSNR values obtained with a higher step size value are still acceptable in the context of a joint scheme as shown in Fig. 8. We note that the image fidelity decreases as the step size value increases. It is even more apparent when analyzing the SSIM values (Fig. 9). We note that the perceptual quality of the compressed and watermarked images at $\Delta_{\mathrm{TCQ}}=2 \Delta$ decreases drastically in comparison with those obtained with other step size values. Figure 10 shows the watermarked and compressed Lena image at $1.6 \mathrm{bpp}$ with step size values in the range of $\Delta / 4$ and $2 \Delta$.

\subsection{Third experimental protocol: watermark robustness study}

In a third round of experiments, the same database of 200 images has been considered to evaluate the robustness of the joint scheme. Four kinds of attacks have been performed: Gaussian filtering attack, Gaussian noise attack, volumetric attack, and JPEG attack similarly to Miller et al. [3]. The bit error rate (BER) is computed for each attack. The BER is the number of erroneous extracted bits divided by the total number of embedded bits. When analyzing the results, the BER values lower than 0.1 are considered. The BER results

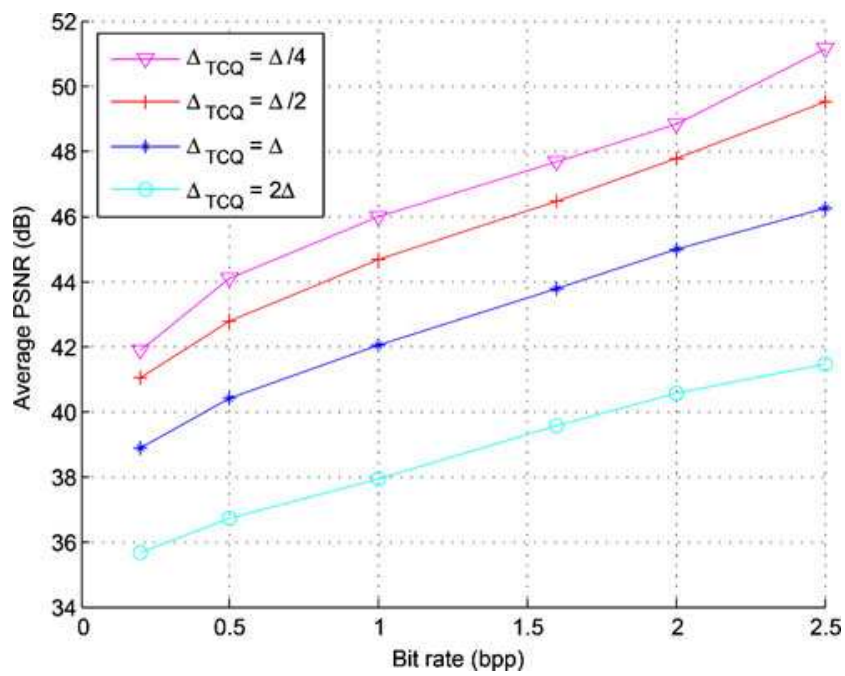

Fig. 8 Average PSNR results obtained by the proposed joint scheme for different step size values on 200 images of size $512 \times 512$ 


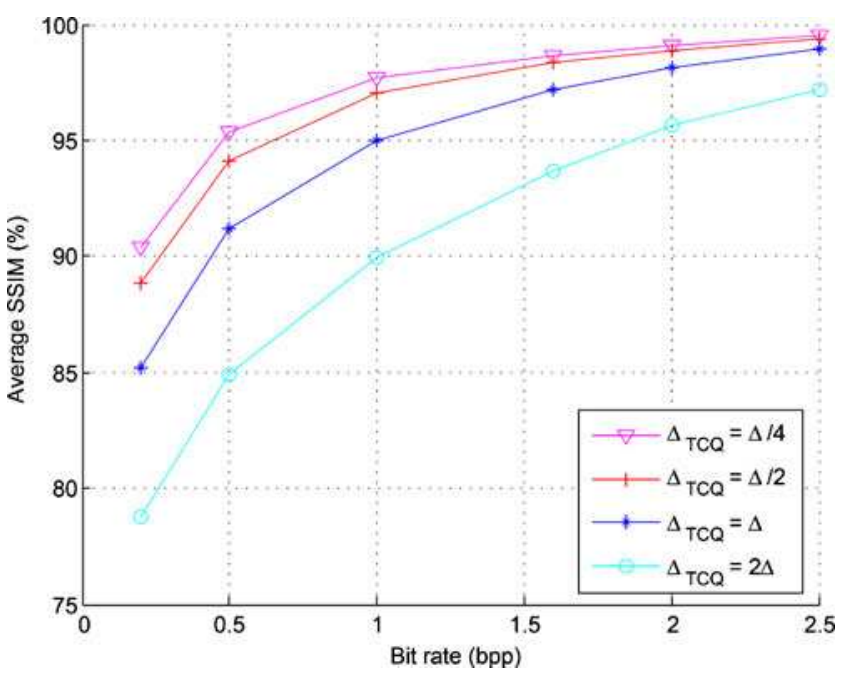

Fig. 9 Average SSIM results obtained by the proposed joint scheme for different step size values on 200 images of size $512 \times 512$

for the four attacks are presented in Figs. 11, 12, 13, and 14. The logarithmic (base 10) scale is used for the $Y$-axis (BER results).

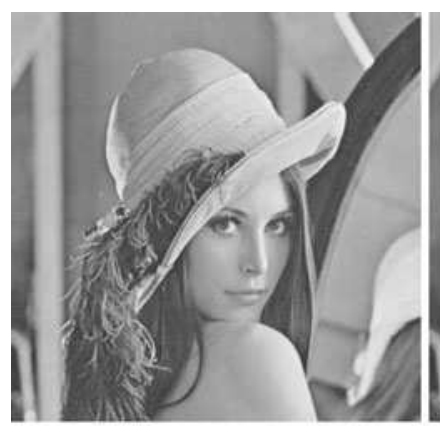

(a)

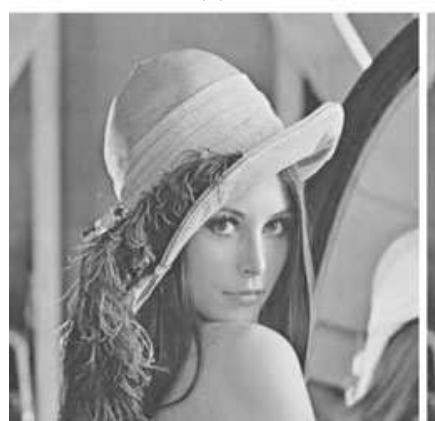

(c)

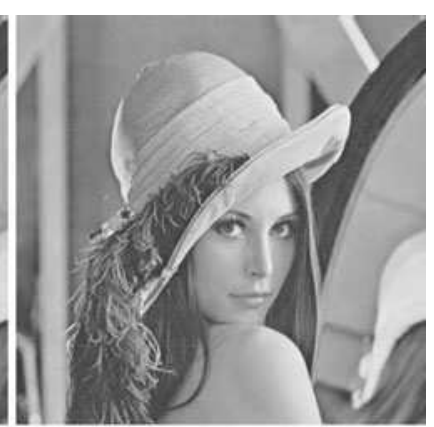

(b)

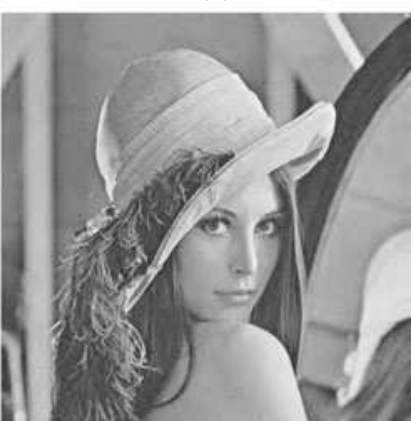

(d)
Fig. 10 Lena image watermarked and compressed with our joint scheme at $1.6 \mathrm{bpp}$ with different step size values: $\mathbf{a} \Delta_{\mathrm{TCQ}}=\Delta / 4$ $(\mathrm{PSNR}=43.09 \mathrm{~dB}, \mathrm{SSIM}=0.9658), \mathbf{b} \Delta_{\mathrm{TCQ}}=\Delta / 2(\mathrm{PSNR}=$ $41.86 \mathrm{~dB}$, SSIM $=0.96021)$, $\mathbf{c}$ watermarking and JPEG2000 compression with $\Delta_{\mathrm{TCQ}}=\Delta(\mathrm{PSNR}=39.53 \mathrm{~dB}, \mathrm{SSIM}=0.9412)$, d watermarking and JPEG2000 compression with $\Delta_{\mathrm{TCQ}}=2 \Delta$ $(\mathrm{PSNR}=36.28 \mathrm{~dB}, \mathrm{SSIM}=0.9010)$

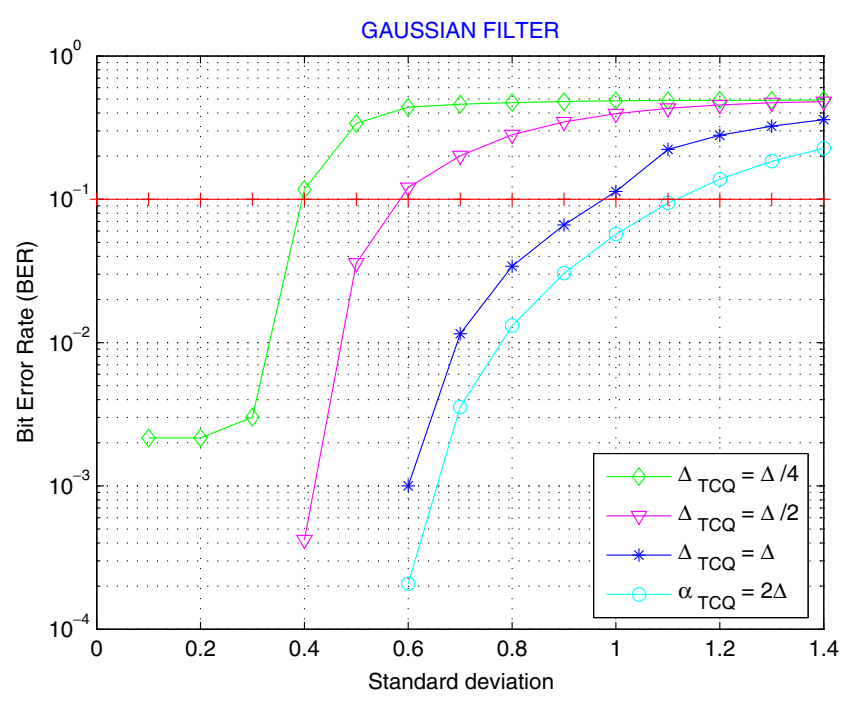

Fig. 11 BER results for filtering attack

The watermarked images are filtered by Gaussian filter of width $\sigma$. The experiment was repeated for different values of $\sigma$, and the BER has been computed. The obtained results are reported in Fig. 11. The watermarks survive filtering effects up to $\sigma=0.3$ for $\Delta_{\mathrm{TCQ}}=\Delta / 4$. As expected, the robustness is improved when the step size value increases. The BER obtained is lower than 0.1 when the joint scheme undergo Gaussian filtering up to: $\sigma=0.5$ for $\Delta_{\mathrm{TCQ}}=\Delta / 2, \sigma=0.9$ for $\Delta_{\mathrm{TCQ}}$ $=\Delta$, and $\sigma=1.1$ for $\Delta_{\mathrm{TCQ}}=2 \Delta$. The BERs for the four values of $\Delta_{\mathrm{TCQ}}$ after an additive white Gaussian noise attack have been measured for different watermark-tonoise ratio as shown in Fig. 12. The joint scheme is not

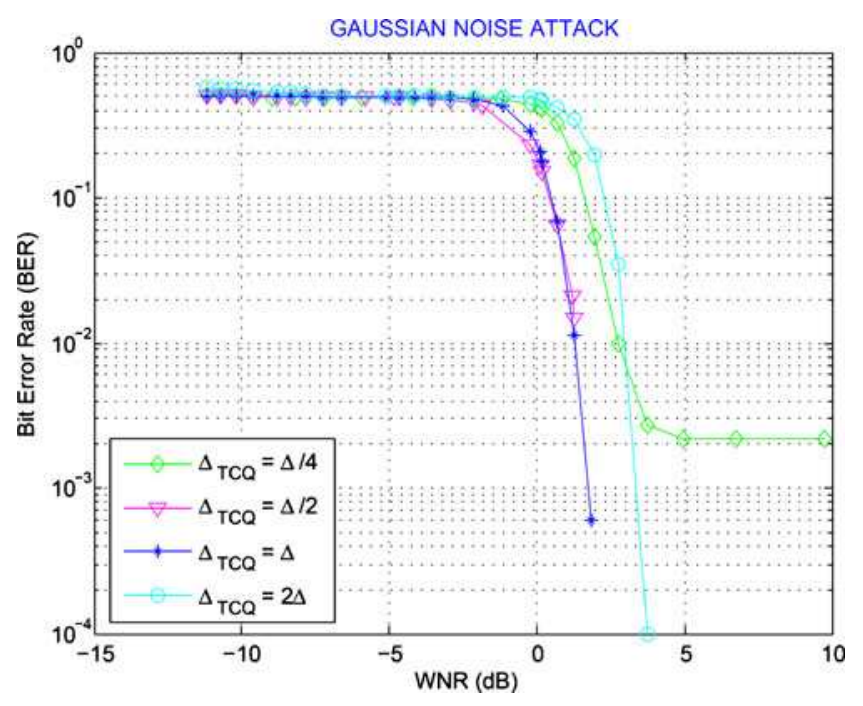

Fig. 12 BER results for Gaussian attack 


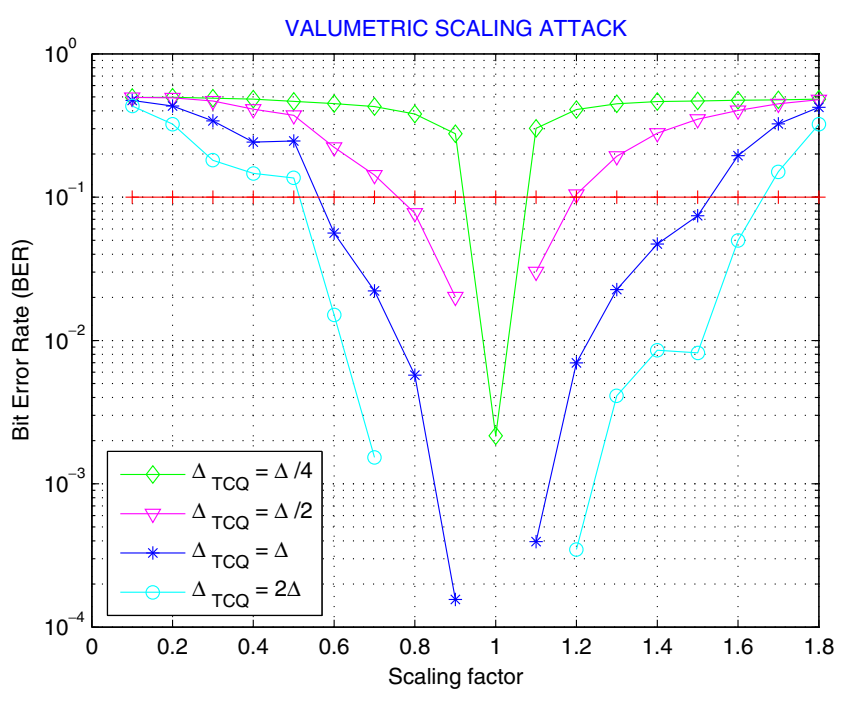

Fig. 13 BER results for scaling attack

very robust to this kind of attack. For $\Delta / 4$ and $\Delta / 2$, the BERs are high but it is more robust for $\Delta$ (up to $\sigma=1$ ) and $2 \Delta$ (up to $\sigma=2$ ).

The results against the volumetric scaling attack are summarized in Fig. 13. The quantized-based watermarking algorithms are recognized to be very sensitive to volumetric scaling (each pixel is multiplied by a constant). As usual, the robustness is better for $\Delta_{\mathrm{TCQ}}$ $=2 \Delta$ : The BER results are under the 0.1 limit when there are changes in scaling with a scaling factor in the range of 0.6 and 1.6. Figure 14 shows the BER results against JPEG attack. We observe that the watermarks are only able to cope with JPEG quality factor up to 80 for $\Delta_{\mathrm{TCQ}}=2 \Delta$. The weak robustness to JPEG attack is inherent to the approach since the coefficients included in the watermarking process are partly high-frequency wavelet coefficients.

In order to analyze the performance of the proposed joint system in terms of robustness, we compare its robustness with that of a conventional watermarking scheme. We use the dirty paper trellis codes [3], which have been proven to achieve high performances with respect to robustness and payload. We use a specific protocol for the DPTC code to be able to make a valid comparison: We perform a JPEG2000 compression attack after watermark embedding and before performing robustness attacks. We fixed the degradation to an average PSNR value of $45 \mathrm{~dB}$ for the two schemes. The payload is fixed to 1 bit embedded in 256 pixels (1,024 bits for $512 \times 512$ images). We use the step size $\Delta_{\mathrm{TCQ}}=\Delta$ when comparing our results with those obtained by DPTC scheme because it provides the best trade off between robustness and visual

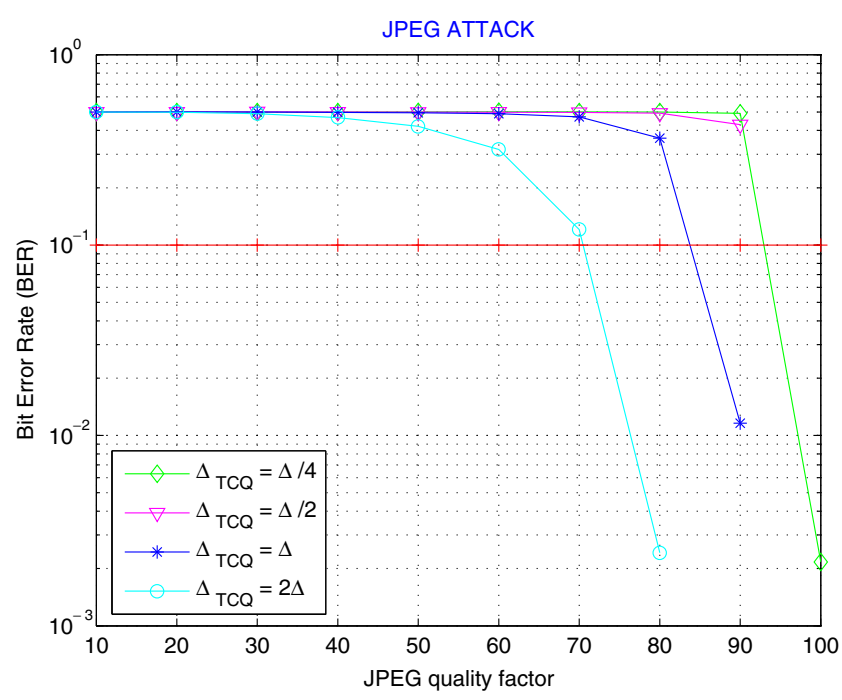

Fig. 14 BER results for JPEG attack

quality. We embed the message in the detail sub-bands of the second to the fourth resolution levels and the rate of the repetition code is $1 / 63$. The compression bitrate is fixed to $2 \mathrm{bpp}$. The experimental results show that DPTC outperforms our joint scheme in terms of robustness for Gaussian noise and JPEG attack. For Gaussian filtering, the robustness is relatively the same as ours, but the BERs obtained by DPTC are smaller than our joint scheme as shown in Fig. 15a. We notice that from $\sigma=0.8$, we obtain better BER values than the DPTC algorithm. For the volumetric scaling, the DPTC results are better than ours for volumetric scaling down. However, we obtain better results for volumetric scaling up than DPTC as shown in Fig. 15b. To sum up, the DPTC scheme provides better robustness results than our joint scheme. The main reason is that DPTC watermarking makes use of both informed coding and informed embedding while our method uses only informed coding. However, DPTC scheme suffers from its CPU computation complexity. Three hours are necessary to watermark an $512 \times$ 512 image with the DPTC algorithm on an Intel dual core $2-\mathrm{GHZ}$ processor while it requires only $2 \mathrm{~s}$ to watermark and compress the same image with our joint scheme.

\subsection{Comparison with other quantization-based joint schemes}

We compare our results with those obtained by the three quantization-based watermarking schemes [1113] of the state-of-the-art to evaluate the performances. The step size value used in the joint scheme is equal 

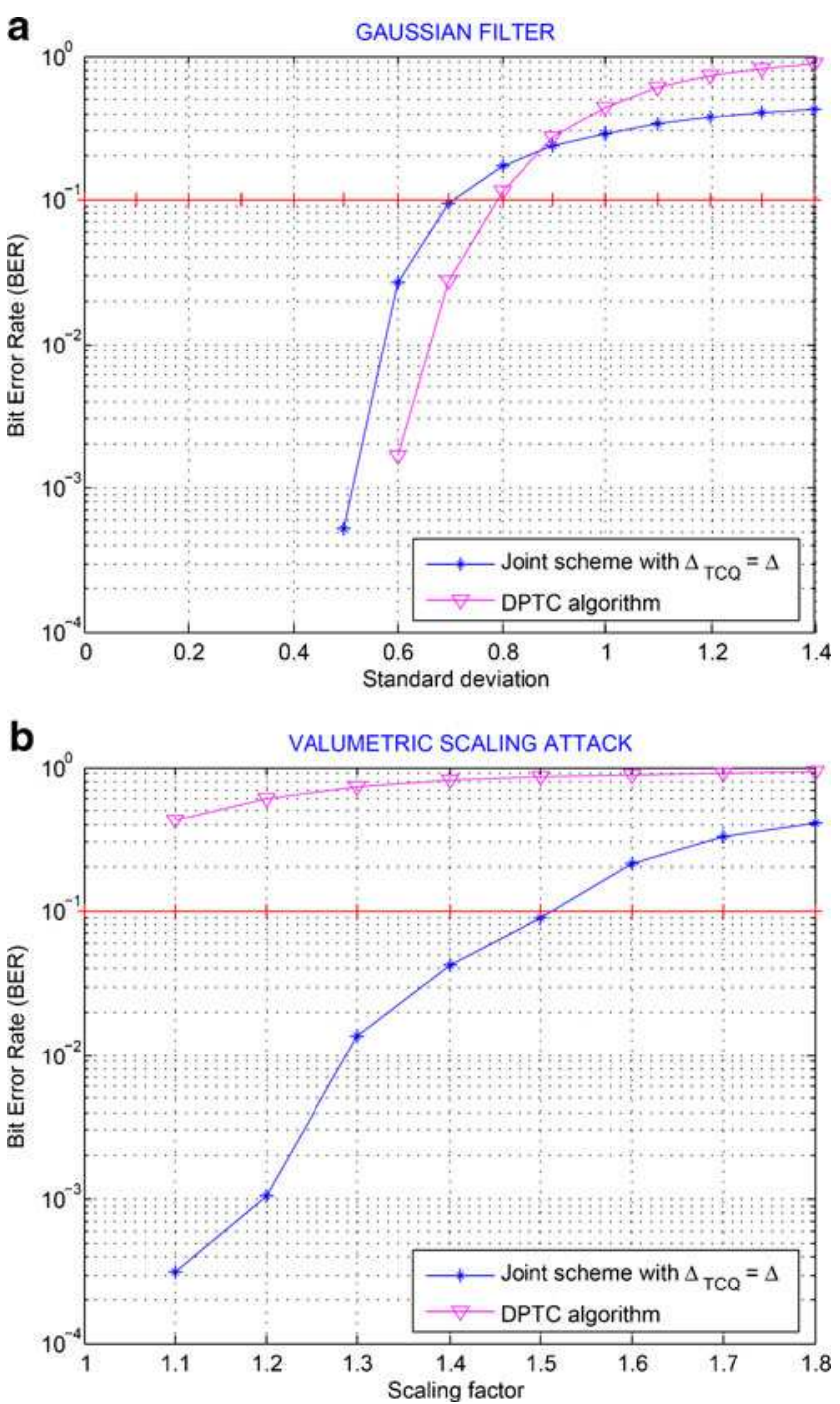

Fig. 15 Robustness performance comparison between our joint scheme and the DPTC scheme for: a Gaussian filter, b volumetric scaling up

to $\Delta$. The first comparison is made between our work and Meerwald algorithm [11]. Meerwald gives PSNR values for the three test images, Lena, Goldhill, and Fishing boat without specifying the target bitrate. The watermark message length is relatively short, about 85 bits for Lena image, 194 bits for Goldhill image, and 383 bits for fishing boat image. For the same payloads and for different bitrates, our joint scheme gives better PSNR values. For Goldhill image, we obtain a PSNR greater than $36 \mathrm{~dB}$ for all the tested bitrates (40.78 dB at 2 bpp, $39.40 \mathrm{~dB}$ at $1 \mathrm{bpp}$, and $36.24 \mathrm{~dB}$ at $0.2 \mathrm{bpp}$ ) while Meerwald method gives a PSNR of $32.09 \mathrm{~dB}$. For fishing boat image, the PSNR values are greater than $37 \mathrm{~dB}(41.07 \mathrm{~dB}$ at $2 \mathrm{bpp}, 41.61 \mathrm{~dB}$ at $1 \mathrm{bpp}$, and $37.43 \mathrm{~dB}$ at $0.2 \mathrm{bpp}$ ) in comparison with $31.45 \mathrm{~dB}$ for Meerwald proposition. The second
Table 3 Comparison of image quality (in terms of PSNR) with Ouled-Zaid et al. scheme [13]

\begin{tabular}{llll}
\hline $\begin{array}{l}\text { Image } \\
\text { test }\end{array}$ & $\begin{array}{l}\text { bitrate } \\
(\mathrm{bpp})\end{array}$ & $\begin{array}{l}\text { Our proposition } \\
\text { PSNR }(\mathrm{dB})\end{array}$ & $\begin{array}{l}\text { Makhloufi et al. } \\
\text { PSNR }(\mathrm{dB})\end{array}$ \\
\hline Lena & 0.6 & 37.80 & 36.55 \\
& 0.4 & 37.30 & 35.23 \\
& 0.2 & 35.30 & 32.39 \\
Bike & 0.6 & 35.46 & 33.88 \\
& 0.4 & 33.15 & 31.58 \\
& 0.2 & 30.90 & 28.11 \\
\hline
\end{tabular}

comparison is made between our work and Schlauweg et al. [12] scheme. Schlauweg et al. proposed a secure authentication scheme based on cryptographic tools. They use a four levels of wavelet decomposition. They mentioned in their paper that the PSNR obtained for Clown and Goldhill images are not good, and they only give the bitrate-PSNR curves for the Clown image. When we compared those curves with ours (for the same payload and the same bitrates), we find that our joint scheme gives similar PSNR values for low bitrates $(<1 \mathrm{bpp})$ and better PSNR values for high bitrates. The last comparison is made between our proposition and Ouled-Zaid et al. [13] scheme. The results of the comparison in terms of PSNR are reported in Table 3 for a payload of 4,096 bits and three levels of wavelet decomposition. We can notice that the PSNR obtained with our joint system are better than their results at different bitrates. When considering the robustness of the watermark against attacks, we cannot perform a valid comparison because none of the other schemes has made extensive experimentations on an image database.

\section{Conclusion and perspectives}

In this work, we propose a joint JPEG2000 coding and informed watermarking scheme based on TCQ which uses duality between the source and the channel coding with side information. The properties of our joint scheme are the following:

- Quantization and watermarking are performed simultaneously. This is the main contribution of this work. In this way, the distortion induced by the insertion of the watermark is minimized.

- Error correcting coding is employed in order to spread the watermark signal on the higher- 
frequency sub-bands because the energy is much lower in comparison with the LL sub-band. It is also used to add redundancy and thus increase robustness.

- High watermarking payloads can be achieved by including as many detail sud-bands as necessary and by adjusting the rate of the error correcting code.

- The step size value of the TCQ quantizers can be set either according to the robustness to be achieved, either in terms of visual quality requirements.

One drawback of the proposed scheme is that some side information is needed at the decoder during JPEG2000 decompression to perform inverse quantization and complete watermark embedding. Experimental investigations demonstrate that this joint scheme is able to achieve good visual quality in terms of PSNR and SSIM. The proposed embedding technique can survive JPEG2000 compression at low bitrates. The watermark robustness against common image attacks have also been studied. It has been noticed that the robustness is improved when a higher quantizer step size value is used. Thus, the selection of the quantizer step size must be done optimally so that the best trade off between robustness and minimum quality degradation should be achieved. The proposed joint scheme can realize high watermarking payloads and can therefore be used in content description and management applications, or in information hiding.

\section{References}

1. Chen B, Wornell G (2001) Quantization index modulation: a class of provably good methods for digital watermarking and information embedding. IEEE Trans Inf Theory 47:14231443

2. Eggers JJ, Bäuml R, Tzschoppe R, Girod B (2003) Scalar costa scheme for information embedding. IEEE Trans Signal Process 51:1003-1019

3. Miller ML, Doerr GJ, Cox IJ (2004) Applying informed coding and informed embedding to design a robust, high capacity watermark. IEEE Trans Signal Process 13(6):792-807

4. Taubman DS, Marcellin MW (2002) JPEG2000 image compression fundamentals standards and practice. Kluwer Academic, Boston

5. ISO/IEC JTCI/SC29 WG1 (2000) JPEG2000 part II final committee draft version 1.0

6. Costa M (1983) Writing on dirty paper. IEEE Trans Inf Theory 29:439-441

7. Dufaux F, Wee S, Apostolopoulos J, Ebrahimi T (2004) JPSEC for secure imaging in JPEG2000. SPIE application of digital image processing, Proc. SPIE. San Jose, USA, pp 319330

8. Fan Y-C, Tsao HW (2007) A dual pyramid watermarking for JPEG2000. Int J High Perform Comput Netw 5:84-96

9. Fan YC, Chiang A, Shen JH (2008) ROI-based watermarking scheme for JPEG 2000. J Circuits Syst Signal Process 27(5):763-774

10. Li K, Zhang X-P (2003) Reliable adaptive watermarking scheme integrated with JPEG2000. International Symposium on Image and Signal Processing and Analysis (ISPA), Proc. ISPA 2003, vol 1. Rome, Italy, pp 117-122

11. Meerwald P (2001) Quantization watermarking in the JPEG2000 coding pipeline. 5th joint working conference on communications and multimedia security, communications and multimedia security issues of the new century, Proc. IFIP TC6/TC11. Darmstadt, Germany, pp 69-79

12. Schlauweg M, Profrock D, Muller E (2006) JPEG2000-based secure image authentication. 8th ACM Multimedia and Security Workshop, Proc. MM\&Sec 2006. Geneva, Switzerland, pp 62-67

13. Ouled-Zaid A, Makhloufi A, Olivier C (2009) Improved QIM-based watermarking integrated to JPEG2000 coding scheme. J Signal Image Video Process 3:197-207

14. Marcellin MW, Fischer TR (1990) Trellis coded quantization of memoryless and Gauss-Markov source. IEEE Trans Commun 38:82-93

15. Forney GD Jr (1973) The Viterbi algorithm. IEEE Trans Inf Theory 61:268-278

16. Braci S, Boyer R, Delpha C (2009) Security evaluation of informed watermarking schemes. 16th IEEE international conference on image processing (ICIP), Proc. ICIP 2009. Cairo, Egypt, pp 117-120

17. Ouled Zaid A, Makhloufi A, Bouallegue A (2007) Wavelet domain watermark embedding strategy using TTCQ quantization. Int J Comput Sci Netw Secur (IJCSNS) 7(6):165-170

18. Le-Guelvouit G (2005) Trellis-coded quantization for public-key watermarking. IEEE international conference on acoustics, speech and signal processing (ICASSP 2005), see the website http://www.gleguelv.org/pub/index.html

19. Chou J, Pradhan SS, Ramchandran K (1999) On the duality between data hiding and distributed source coding. Annual Asilomar conference on signals systems and computers, Proc. AACSSC 1999, vol 2. Pacific Grove, USA, pp 1503-1507

20. Esen E, Alatan AA (2004) Data hiding using trellis coded quantization. IEEE international conference on image processing (ICIP), Proc. ICIP 2004, vol 1. Singapore, pp 59-62

21. Wang X, Zhang X-P (2007) Generalized trellis coded quantization for data hiding. IEEE international conference on acoustics, speech and signal processing (ICASSP), Proc. ICASSP 2007, vol 2. Honolulu, Hawaii, USA, pp 269-272

22. Fischer TR, Wang M (1992) Entropy-constrained trelliscoded quantization. IEEE Trans Inf Theory 38:415-426

23. Schuchman L (1994) Dither signals and their effect on quantization noise. IEEE Trans Commun Technol (COM) 12:162_165

24. Pérez-Freire L, Pérez-Gonzalez F (2008) Security of Latticebased data hiding against the watermarked-only attack. IEEE Trans Inf Forensics Security 3(4):593-610

25. Cayre F, Fontaine C, Furon T (2005) Watermarking security: theory and practice. IEEE Trans Signal Process, Special Issue on Content Protection 53:3976-3987 